\title{
Reduction and Fixation of Unstable Slipped Capital Femoral Epiphysis: A Case Report Abdulhamid Talal Alghamdi
}

Department of Orthopedic Surgery, Al Noor Specialist Hospital, Makkah, Saudi Arabia

Corresponding author: Abdulhamid Talal Alghamdi, Mobile: +966500323870, E-Mail: dr.atghamdi@ hotmail.com

\begin{abstract}
Background: Slipped capital femoral epiphysis, is a common condition of the proximal femoral physis that leads to slippage of the epiphysis relative to the metaphysis. Diagnosis can be confirmed with radiographs of the hip. Treatment is usually percutaneous screw fixation.

Objective: To study the outcome and complications of closed reduction and internal fixation by percutaneous screw fixation in unstable slipped capital femoral epiphysis.

Patient and methods: Our case report is 11 years old obese male patient with history of progressive right hip pain for 10 days prior to presentation to the hospital. BMI was $36.1 \mathrm{~kg} / \mathrm{m}^{2}$. Patient presented with right hip pain and inability to bear weight, radiological evaluation confirmed right hip unstable slipped capital femoral epiphysis that required emergency in situ fixation

Result: close follow up of the patient to 6 months, patient regain full functional activity, he achieved a complete range of motion of the right hip with good outcomes and with no complications. The goal in such injuries is to achieve a stable hip joint with gradual rehabilitation to reach a good functional outcome

Conclusion: Slipped capital femoral epiphysis occurs in adolescent age group, more affecting male and obese patient, and they are idiopathic in the majority of cases. The goal of treatment in such patients is to achieve a stable hip joint with gradual rehabilitation to reach the optimal functional outcomes
\end{abstract}

Keywords: Slipped capital femoral epiphysis, Adolescent hip problem, Klein’s line.

\section{INTRODUCTION}

Slipped capital femoral epiphysis (SCFE) is defined as a posterior and inferior slippage of the proximal femoral epiphysis (femoral head) on the metaphysis (femoral neck), occurring through the epiphyseal growth plate during the early adolescent growth spurt ${ }^{(1)}$.

SCFE has been found to affect children aged 9 to 16 years in the United States at a rate of 10.80 cases per 100,000 children ${ }^{(2)}$. In a retrospective review, investigators found that $81.1 \%$ of children with SCFE had a BMI above the $95^{\text {th }}$ percentile (i.e. clinically obese) compared to only $41.3 \%$ of controls ( $\mathrm{P}<$ $0.0001)^{(3)}$.

The obesity had the effect of increasing the weight and cause shearing force across the growth plate of proximal femoral physis leading to progressive slippage of femoral head posterior and inferior in direction to weight-bearing force ${ }^{(3)}$.

This case report presents the initial assessment, diagnosis, efficacy of the closed reduction_and screw fixation method and post-operative follow up, reporting complications if found in patient with a SCFE. Therefore, this study aimed to evaluate the efficacy of the used fixation and treatment methods.

\section{CASE REPORT}

11 years old obese male patient not known to have any medical or surgical past history, came to the hospital with a complaint of severe pain on weight bearing of the right hip after sustaining a very minimal fall about one week ago, that classifies the slip as unstable. The patient gave a history of mild right hip pain for approximately 4 month, making the presentation taken a form of acute on top of chronic. He didn't report left hip pain, he denied any radiation exposure, family history was insignificant.

Physical exam revealed a well-developed, wellnourished child, $158 \mathrm{~cm}$ height and weighed $90 \mathrm{~kg}$, The resultant BMI was $36.1 \mathrm{~kg} / \mathrm{m}^{2} \mathrm{a}$. slight antalgic lean to the left, on supine examination, leg length discrepancy noted with right leg was short by about 2 $\mathrm{cm}$ and externally rotated. The right hip joint ROM was restricted and very painful in passive flexion and internal rotation.

Patient had positive Drehmann sign (obligatory external rotation while passive flexion applied on the hip), positive FABER-Patrick's test (Flexion, Abduction and External Rotation of the hip produce pain or restriction of motion). Supine leg length testing using Allis' sign revealed no gross anatomical leg length discrepancy. 

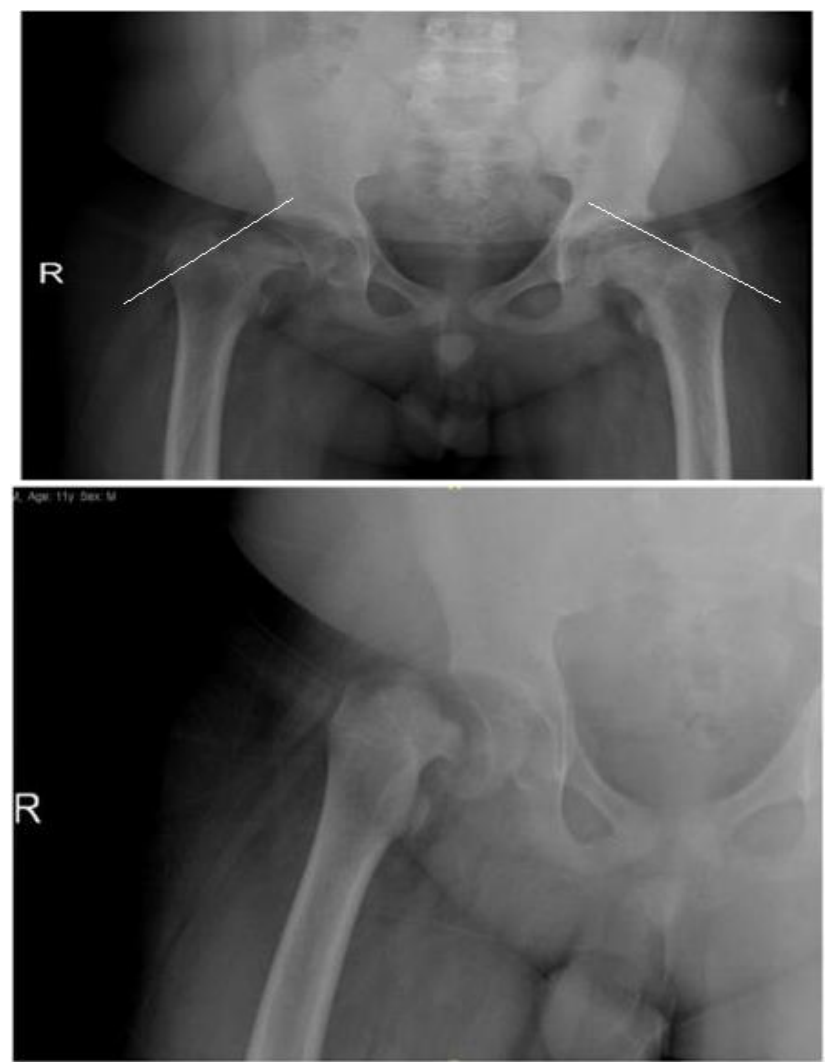

Figure (1): Anteroposterior and Frog-leg radiographs reveal the amplitude of the slippage.

AP and Frog-leg radiographs were then taken at the emergency (figure 1). Klein's line (line drawn along superior border femoral neck) indicated slippage and displacement of femoral epiphysis in relation to the femoral neck of right hip, as compare to left side witch revealed intersection of the Klein's line to femoral capital physis. This displacement was more clearly seen in frog lateral radiography.

In frog lateral view hip indicated a severe slip of $>50 \%$ of the left hip (according to the Southwick anteroposterior angle).

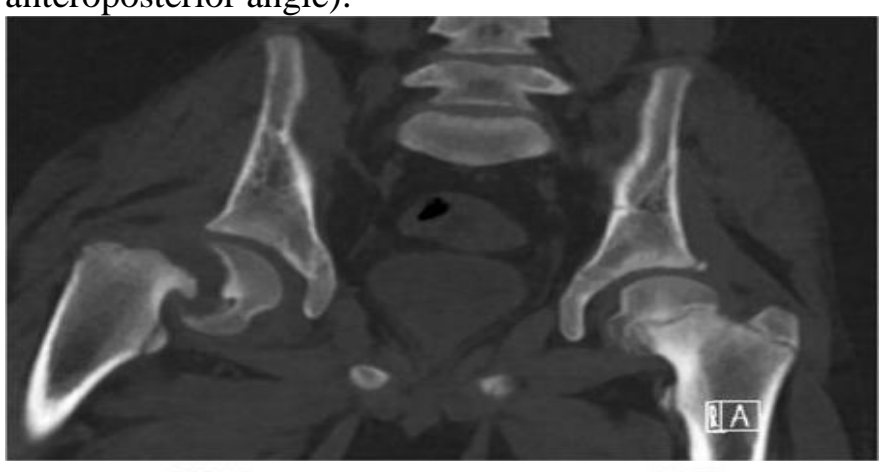

RIGHT

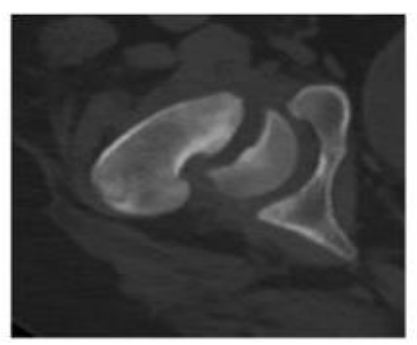

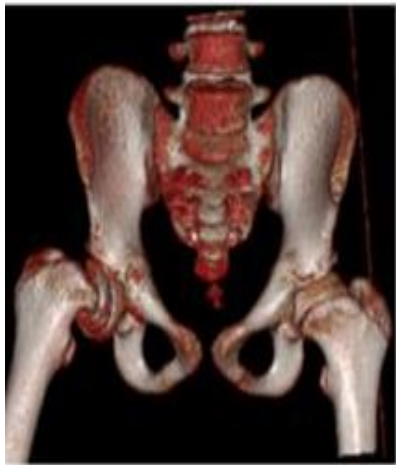

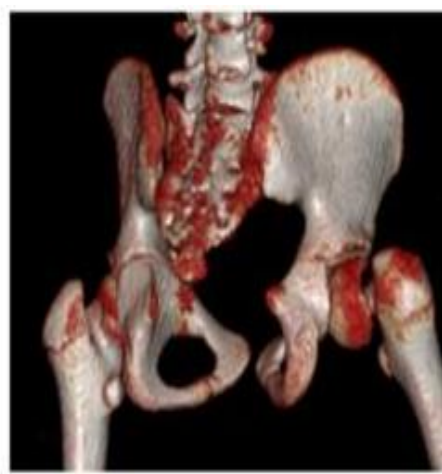

Figure (2): CT scan of the pelvis.

Further radiological evaluation was done, including CT scan of the pelvis (figure 2), as it showed clear physeal slippage and displacement of the proximal femoral physis to the posterior-inferior direction in relation to the neck of right hip. The difference can be detected easily by comparing it to the left hip,

Initial laboratory tests included complete blood count, basic chemistries, serum testosterone, thyroid function test and renal function test were ordered to exclude risk factors. The results of these tests were within normal level.
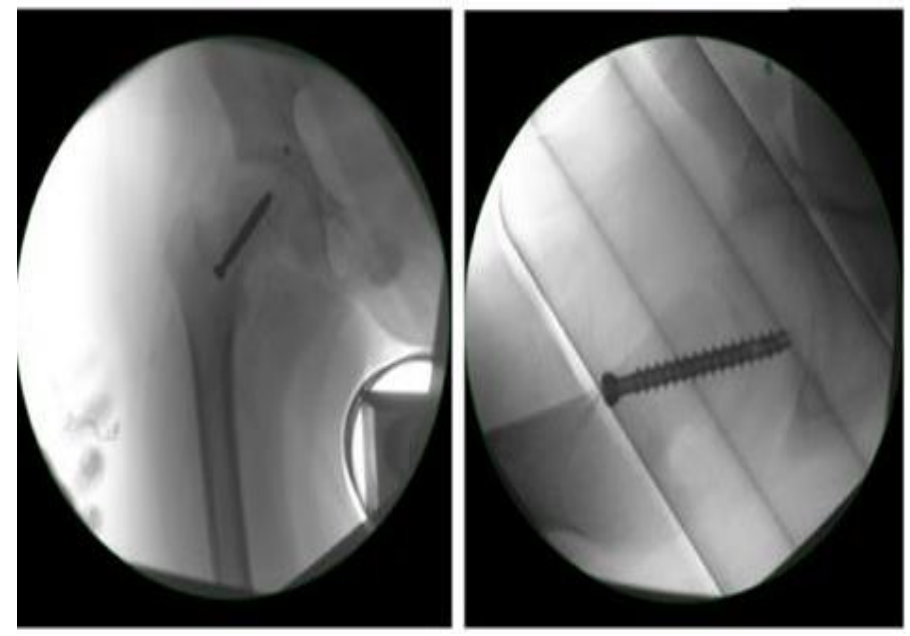

Figure (3): Right hip x ray shows postoperative closed reduction and percutaneous screw fixation.

Then, the patient was admitted and underwent right hip closed reduction on traction table until the femoral head was reduced, and percutaneous fully threaded cancellous screw was used to fix the femoral head in its acceptable position (figure 3).

Postoperatively, the patient was fully recovered and was discharged home three days later. Postoperative instructions were non weight-bearing on right lower limb and follow-up with orthopedic pediatric clinic. On early follow-up, no complications were noted. He progressed to partial weight bearing on the right lower extremity at 6 weeks. Three months after the initial surgery, the patient was tolerating full weight bearing on the right hip without assistance. 

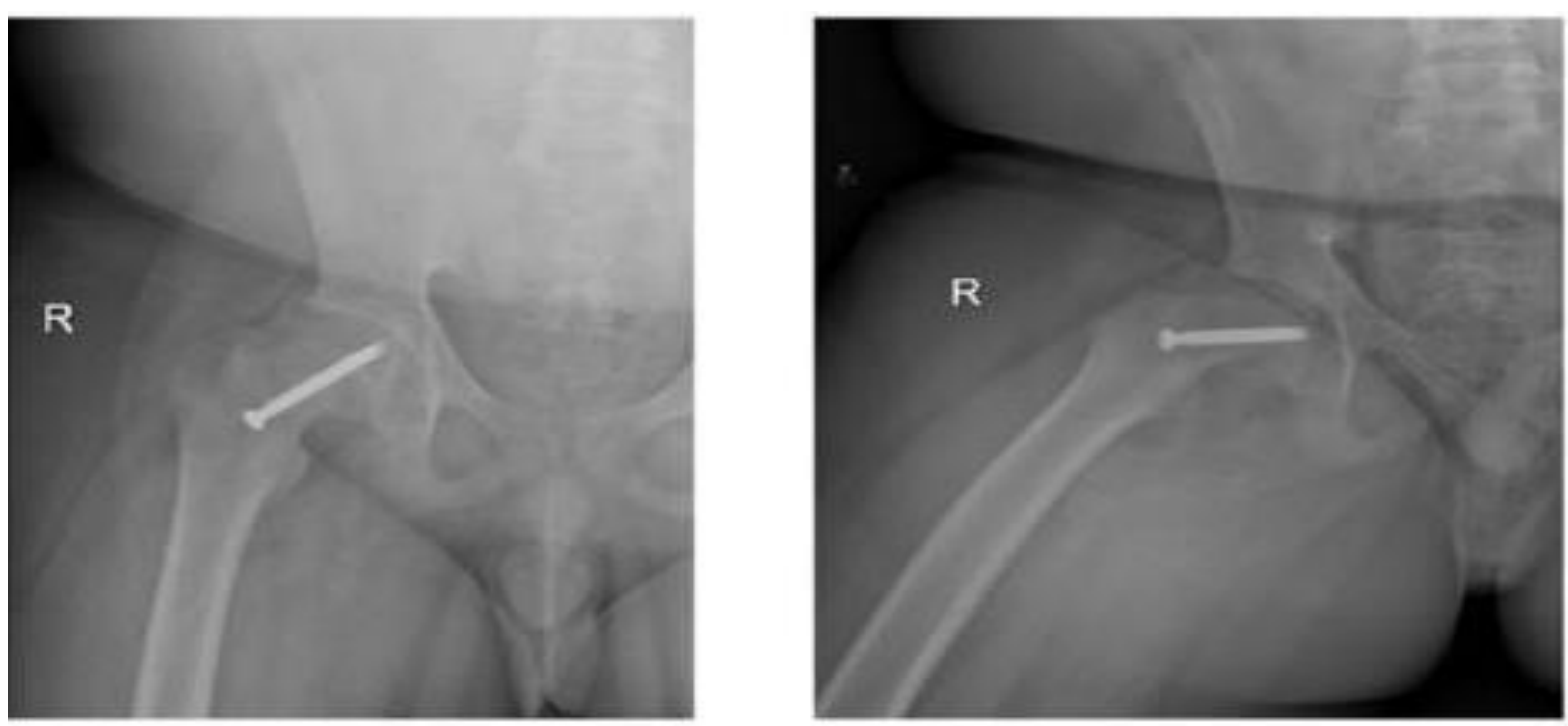

Figure (4): 6 months postoperative AP and frog lateral X-rays.

In (figure 4), anteroposterior and frog lateral radiography of the patient right hip on 6 months follow up postoperatively revealed that the patient had a stable hip with no clear sign of osteolysis, screw cut out or avascular necrosis. Complete range of motion of the right hip with no major restrictions and no pain or limping was noted.

\section{Ethical approval:}

An approval of the study was obtained from Al Noor Specialist Hospital, (Makkah, Saudi Arabia) Academic and Ethical Committee. Patient signed an informed written consent for acceptance of the operation and for publication of this case report. This work has been carried out in accordance with the Code of Ethics of the World Medical Association (Declaration of Helsinki) for studies involving humans.

\section{DISCUSSION}

The patient who presents with SCFE must be thoroughly evaluated. The typical management for SCFE once it is diagnosed is surgical reduction by pinning the slipped femoral epiphysis to the metaphysis in situ ${ }^{(1,4)}$. The use of manipulation in conjunction with surgical treatments for SCFE is contentious in the medical studies, especially in situations of acute slippage ${ }^{(5)}$.

Lim et al. ${ }^{(5)}$ reported a total of 24 cases, acute or acute-on-chronic SCFE underwent preoperative traction, 13 of them followed by in situ screw fixation, and the remaining 11 , manipulation with screw fixation. There were 2 treatment failures and one poor outcome in patients with acute SCFE ( 2 had avascular necrosis and one had slight pain with mild limitation of hip movement). As a conclusion the risk of developing avascular necrosis was significantly higher in patients with acute rather than acute-onchronic or chronic slips. Patients with acute or acuteon-chronic SCFE can be safely managed with straight-leg traction for up to 6 days, followed by in situ screw fixation without manipulation. Manipulation is not recommended in such slips, due to higher avascular necrosis risk. Conversely, research has also shown that preoperative manipulation can be safe, and when an acute slip is reduced early $(<24$ hours), the AVN risk may actually decrease ${ }^{(6)}$.

In our case the injury was unstable, that reduced easily by gentle traction on traction table, as the patient underwent the surgery within one day from admission.

Post-operative management of patients with SCFE typically non-weight bearing of the involved hip as the patient remains in crutches for four to six weeks ${ }^{(1,4)}$.

Surgeons often prescribe gentle toe touching exercises for the patient as well while in crutches followed by a gradual return to normal daily activities after the minimum four to six weeks-period.

In our case starting with toe weight bearing was challenging as the patient had obesity preventing him from taking the advantage of early rehabilitation and early return to function

The biomechanical studies have shown that there is a $33 \%$ increase in the construct strength when 2 screws are utilized but no additional proportional increase with 3 or more screws. However, the use of single percutaneous screw had advantage of minimizing the iatrogenic injury with the multiple screw approach ${ }^{(7)}$.

The chronicity pattern in our case, which resulted in a complete dislocation of the femoral head as well as technical way of operation that use the traction table for reduction decreases the chances of adding more risk of avascular necrosis and jeopardizing the blood supply of the femoral head.

\section{CONCLUSION}


Adolescents with unstable SCFE should be managed with caution, as they are at significant risk to develop avascular necrosis of the joint. However, the optimal time when to fix the joint, the use of traction to gain better reduction that improves the overall outcomes and single vs multiple screws used for fixation in such cases remains controversial.

Careful rehabilitation program for every case to maximize the functional outcomes and minimize the risks of complications had to be considered.

Further clinical studies and researches are still needed regarding operative management of SCFE to guide the best clinical and functional outcomes in the future.

In conclusion, educating the patient and the family, close observation, and medical management are vital to the comprehensive treatment of this condition.

\section{ACKNOWLEDGMENT}

I would like to thank the patient for his cooperation and for his approval for making of this case report.

Conflict of interest: The author declares no conflict of interest.

Funding sources: The author has no funding to report.

\section{REFERENCES}

1. Loder $R$ (1998): Slipped capital femoral epiphysis. Am Fam Physician, 57 (9): 21352142.

2. Lehmann $\mathrm{C}$, Arons R, Loder $\mathrm{R}$ et al. (2006): The epidemiology of slipped capital femoral epiphysis: an update. J Pediatr Orthop., 26 (3): 286-290.

3. Manoff E, Banffy M, Winell J (2005): Relationship between Body Mass Index and slipped capital femoral epiphysis. J Pediatr Orthop., 25 (6): 744-746.

4. Aronsson D, Loder R, Breur G et al. (2006): Slipped capital femoral epiphysis: current concepts. J Am Acad Orthop Surg., 14 (12): 666679.

5. Lim Y, Lam K, Lim $K$ et al. (2007): Management outcome and the role of manipulation in slipped capital femoral epiphysis. J Orthop Surg (Hong Kong), 15 (3): 334-338.

6. Peterson M, Weiner D, Green N et al. (1997): Acute slipped capital femoral epiphysis: the value and safety of urgent manipulative reduction. $\mathrm{J}$ Pediatr Orthop., 17 (5): 648-654.

7. Karol L, Doane R, Cornicelli S et al. (1992): Single versus double screw fixation for treatment of slipped capital femoral epiphysis: a biomechanical analysis. J Pediatr Orthop., 12: 741-745. 